\title{
Apport de l'immunologie à la prise en charge diagnostique et thérapeutique des glomérulonéphrites extramembraneuses
}

Charline Miota, ${ }^{a, b}$, Caroline Polia,b, Céline Beauvillaina ${ }^{a, b}$, Pascale Jeannin ${ }^{a, b}$, Gilles Renier $^{a, c}$, Jean-François Subra ${ }^{b, d}$, Alain Chevailler $^{a, b, \star}$

\section{Résumé}

La glomérulonéphrite extra-membraneuse idiopathique peut désormais être qualifiée de maladie autoimmune glomérulaire. Un certain nombre d'autoanticorps a été décrit contre des composants glomérulaires ou contre des protéines alimentaires usuelles. Certains de ces autoanticorps, tel que les anticorps anti-PLA2R et les anticorps anti-THSD7A sont utiles pour suivre l'activité de la maladie et la réponse au traitement. Il existe certainement une susceptibilité génétique pour l'apparition de la maladie. La meilleure connaissance de la physiopathologie est le support rationnel à de nouvelles approches thérapeutiques en cours d'évaluation.

Anticorps anti-PLA2R - anticorps anti-THSD7A glomérulonéphrite extra-membraneuse.

\section{Introduction}

La glomérulonéphrite extra-membraneuse (GEM) est la cause la plus fréquente de syndrome néphrotique chez l'adulte. Le diagnostic repose sur l'histologie rénale avec étude en immunofluorescence directe qui révèle des dépôts granuleux d'immunoglobulines (IgG4, IgG1) et de complément visible sur le versant externe de la membrane basale glomérulaire responsables de l'aspect épaissi de la membrane basale en microscopie optique; il n'est pas mis en évidence de prolifération ou d'infiltration cellulaire [1]. Différents types de mécanismes sont

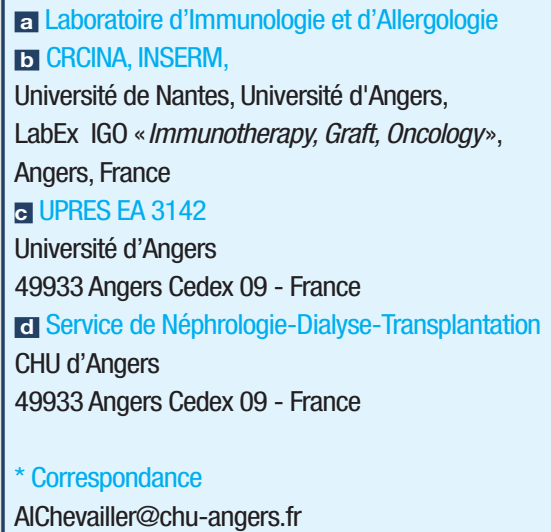

\begin{abstract}
SUMmaRY
Idiopathic membranous nephropathy, recent advances

From now on, idiopathic membranous nephropathy could be ascertained as autoimmune disease, since autoantibodies were isolated against glomerular components, or even against usual dietary antigens. Some of them, like anti-PLA2R antibodies or anti-THSD7A are useful to monitor the disease activity and to follow the treatment efficacy. Genetic polymorphisms of PLA2R gene may be the underlying cause of idiopathic membranous nephropathy. This translational research opens a new field to develop innovative targeted therapies.
\end{abstract}

Anti-PLA2R antibody - anti-THSD7A antibody membranous nephropathy

susceptibles d'aboutir à ces lésions, que l'on regroupe depuis longtemps en GEM primaires ou idiopathiques, et GEM secondaires, selon la nature de l'atteinte rénale, isolée ou associée à une entité nosologique extra-rénale clairement distincte. Le rôle néphritogène des complexes immuns est bien établi avec, pour les formes secondaires des antigènes exogènes, et pour les formes idiopathiques des antigènes soit allogènes, soit autoantigènes [2].

Le spectre des GEM chez l'homme doit être reconsidéré au regard des données les plus récentes et de la mise en évidence d'antigènes cibles responsables d'une réponse immune. Quelques très rares cas de GEM néonatale par alloimmunisation contre l'endopeptidase neutre ont été décrits. Le rôle d'antigènes alimentaires (sérum albumine cationique) ou environnementaux est aussi reconnu, dans la petite enfance et chez l'adulte. Chez l'adulte, au cours des GEM secondaires on sait que certains déterminants du virus de l'hépatite $B$ sont en cause, mais c'est dans le cadre des GEM auto immunes que les progrès les plus notables ont été réalisés ces dernières années. Chez le patient transplanté rénal, la récidive de ces GEM autoimunes est bien documentée, mais peut survenir aussi une GEM «alloimmune».

Dans approximativement $20 \%$ des cas, les GEM sont secondaires: les sous-classes d'lgG déposées sont majoritairement lgG2 et IgG3. Les exoantigènes en cause sont des 
Tableau I- Étiologies des glomérulonéphrites extra-membraneuses (GEM) secondaires.

\begin{tabular}{|c|c|}
\hline \multicolumn{2}{|r|}{ GEM secondaires } \\
\hline $\begin{array}{l}\text { - Infections } \\
\text { - Hépatite virale B (HVB) } \\
\text { - Paludisme } \\
\text { - Syphilis } \\
\text { - Lèpre } \\
\text { - Filarose } \\
\text { - Kyste hydatique } \\
\text { - Schistosomiase }\end{array}$ & $\begin{array}{l}\text { - Médicaments } \\
\text { - Antiinflammatoires non stéroïdiens } \\
\text { (NSAID) } \\
\text { - Sels d'or } \\
\text { - Pénicillamine } \\
\text { - Tumeurs solides } \\
\text { - Transplantation rénale } \\
\text { et greffe de moëlle } \\
\text { - Alloimmunisation } \\
\text { - Ag mineur histocomptabilité (HC) }\end{array}$ \\
\hline $\begin{array}{l}\text { - Maladies autoimmunes } \\
\text { - Lupus érythémateux sys } \\
\text { - Polyarthrite rhumatoïde } \\
\text { - Sjögren } \\
\text { - Thyroïdopathie }\end{array}$ & $\begin{array}{l}\text { (MAI) } \\
\text { émique (LES) } \\
\text { PR) }\end{array}$ \\
\hline
\end{tabular}

molécules cationiques (nucléosomes, antigène du virus de l'hépatite B) capables d'interagir avec les structures anioniques du glomérule (théorie de l'antigène planté), ou, de manière alternative, des complexes immuns circulants peuvent se dissocier et se reformer dans l'espace sous-épithélial. Les différentes étiologies des GEM secondaires sont reportées dans le tableau / [3].

Quatre-vingt pour cent des GEM sont donc primaires, touchant préférentiellement les hommes (sex-ratio de $2: 1$ ) de 30 à 50 ans [4] et dont on sait désormais que pour la grande majorité elles sont des maladies auto-immunes spécifiques d'organe dans lesquelles des auto-anticorps circulants se lient à des antigènes exprimés à la surface des podocytes. C'est en 2009 qu'un premier auto-antigène podocytaire a été identifié, le récepteur de la phospholipase $A 2$ de type $M$ (PLA2R) [5], et que des anticorps anti-PLA2R ont été mis en évidence dans le sérum de patients ayant une GEM idiopathique en utilisant une technique d'immunoempreintes. Leur détection s'est simplifiée en 2010 avec l'apparition d'une technique commerciale d'immunofluorescence indirecte sur cellules transfectées, permettant une plus large étude de ce marqueur suivi par la disponibilité d'un test ELISA. Depuis 2012 et la parution de notre revue sur les actualités de néphro-immunologie [6], une deuxième cible auto-antigénique a été individualisée, la THSD7A (pour thombospondine type 1 domain-containing $7 A$ ), identifié de manière collaborative avec les mêmes outils $[7,8]$.

Traditionnellement, la distinction entre GEM primaire et secondaire repose sur une démarche intégrative clinique et anatomopathologique. L'irruption de tests commerciaux pour rechercher les auto-anticorps [9] a conduit certains auteurs à proposer de nouveaux algorithmes reposant sur l'adjonction des tests immunologiques dans la démarche anatomoclinique [10, 11].

Après avoir brièvement décrit les cibles auto-antigéniques et les tests diagnostiques, cette revue se propose de répondre aux questions suivantes:

i) est-ce que la recherche des anticorps anti-PLA2R et antiTHSD7A permet de s'affranchir de la ponction-biopsie rénale? ii) est-ce que la recherche des anticorps anti-PLA2R et antiTHSD7A permet de clairement distinguer GEM primaire et GEM secondaire, et donc de s'affranchir d'un lourd bilan étiologique?

iii) est-ce que le titre des auto-anticorps a une valeur pronostique et peut donc faciliter la décision de commencer, d'adapter ou d'interrompre un traitement immunosuppresseurlourd? iv) est-ce que la positivité de la recherche des auto-anticorps peut faciliter la prédiction, la détection et le traitement des récidives post-transplantation? Parce que la découverte des anticorps anti-THSD7A est plus récente, le corpus de données bibliographiques est plus important pour les anticorps anti-PLA2R.

\section{Auto-antigènes et auto-anticorps}

\subsection{Le récepteur de la phospholipase $A 2$ de type $M$ comme cible autoantigénique}

\subsubsection{Identification de la cible}

En 2009, Beck et al [5] ont identifié le PLA2R comme cible autoantigénique dans une étude portant sur une cohorte de 37 patients avec $70 \%$ de positifs. La technique utilisée était une technique d'immunoempreintes en conditions non dénaturantes d'un extrait de glomérules humains normaux visualisant une bande de $185 \mathrm{kDa}$, identifiée par spectrométrie de masse comme étant le PLA2-R et confirmée par immunoprécipitation avec du PLA2-R recombinant. Le PLA2-R est présent dans les podocytes des glomérules humains et dans les dépôts de GEM idiopathiques où il colocalise avec les IgG4. Les IgG éluées des biopsies de GEM idiopathiques reconnaissent le PLA2-R.

Le PLA2R est un récepteur transmembranaire de type I qui appartient à la famille des mannose récepteurs, avec le récepteur Ca-dépendant du mannose-6-phosphate, le C-type mannose récepteur 2, le récepteur DEC205 des cellules dendritiques et le récepteur FcRY. Par recyclage entre la membrane plasmique et le compartiment endosomal, ces récepteurs permettent l'internalisation de ligands extra-cellulaires. Ils peuvent adopter deux configurations: étendue ou repliée, ce qui conditionne, pour le PLA2R, l'accessibilité des épitopes reconnus par les anticorps des patients [12]. Son ligand est la phospholipase A2, enzyme lipolytique qui clive les acides gras liés aux glycérophospholipides de membrane. Le PLA2R est une glycoprotéine de $185 \mathrm{kDa}$ avec une longue séquence extra-cellulaire $\mathrm{N}$ terminale, une seule portion transmembranaire et une courte région intra-cytoplasmique $\mathrm{C}$ terminale. La portion intracellulaire possède un domaine d'internalisation. La région extracellulaire est constituée d'un domaine $\mathrm{N}$ terminal riche en cystéine, d'un domaine homologue à la fibronectine de type II et de huit domaines homologues aux lectines de type C (CRD1 à 8). Le domaine CRD5 est impliqué dans l'interaction avec le ligand [13]. Le PLA2R est exprimé, entre autres, sur les podocytes, où sa fonction n'est pas formellement connue. II n'existe cependant pas de preuve directe de la fixation de la PLA2 à son récepteur. L'épitope dominant se situe dans le domaine de type ricine 
$\mathrm{N}$-terminal [14]. Le rôle principal du PLA2R est de soustraire la PLA2 de la circulation, régulant ainsi ses effets biologiques [15].

II a été retrouvé une susceptibilité génétique aux GEM idiopathiques avec une forte association avec l'haplotype HLA-B8/DR3 dans les populations européennes [4]. Le gène du PLA2R présente également un polymorphisme de susceptibilité de type SNP (single nucléotide polymorphisme). Dès 2010, Liu et al [16] pointaient le rôle possible d'une susceptibilité génétique dans la survenue de la GEM idiopathique sous la forme d'une association préférentielle de certains variants de PLA2R, ce qu'ont confirmé Stanescu et al [17] avec aussi une association préférentielle à la chaîne alpha de HLA-DQA1 [12]. L'odds ratio pour les sujets homozygotes pour ces deux facteurs de risque est de 80 .

De plus un mimétisme moléculaire pourrait participer à la rupture de tolérance puisqu'il a été montré des homologies de séquences entre des peptides issus du PLA2R et une enzyme de la paroi de bactéries du genre Clostridium. Laconjonction de plusieurs facteurs, génétiques (polymorphisme du PLA2R et de HLA-DQA1) et environnementaux (infection bactérienne) serait nécessaire pour l'apparition de la réponse anti-PLA2R. Reste à expliquer une situation, somme toute assez fréquente en pathologieauto-immune: pourquoi vis-à-vis d'un auto-antigène largement distribué comme le PLA2R, la rupture de tolérance ne se traduit-elle que par une maladie rénale [15]?

L'absence de PLA2R dans les glomérules de rongeur empêche la réalisation de modèles expérimentaux par injections d'anticorps humains à l'animal pour reproduire une néphrite de type Heymann, qui est le modèle des GEM [6]. Le recours à des souris transfectées pour le gène du PLA2R sera peut-être la solution [18].

Alors que la destruction podocytaire semble médiée par le complément, les données de l'immunofluorescence (dépôts d'IgG4 et absence de C1q) plaident contre une activation du complément par la voie classique. La présence de C4b est plus en faveur d'une activation de la voie des lectines que de la voie alterne [3]. Les autoanticorps de classe IgG4 pourraient être dépourvus de galactose terminal et pourraient donc fixer la mannose binding lectine, qui enclenche la voie d'activation des lectines [4].

L'insertion du complexe d'attaque membranaire dans la membrane plasmique des podocytes, étape ultime de l'activation du complément, aboutit à l'activation de ces derniers avec production de radicaux libres d'oxygène, désorganisation de la membrane basale glomérulaire, surproduction de composants de la matrice extracellulaire, et au final altération de la filtration glomérulaire [15]. Deux hypothèses sont donc au moins possibles pour la rupture de tolérance:

i) pour la première, l'interaction directe de peptides issus du PLA2R libérés par les podocytes exprimant un allèle de susceptibilité de HLA-DQA1 déclenche la réaction auto-immune;

ii) pour la seconde un mimétisme moléculaire avec des antigènes microbiens ou environnementaux conduit à la production d'anticorps anti-PLA2R chez des sujets qui ont l'allèle de susceptibilité HLA-DQA1 [5].

\subsubsection{Les tests diagnostiques}

Les tests d'immunoempreintes utilisés pour la caractérisation des cibles auto-antigéniques, de par leur caractère artisanal, ne sont pas adaptés à une utilisation quotidienne en pratique diagnostique. Le test d'immunofluorescence indirecte (IFI) sur cellules transfectées est semi-quantitatif et permet le suivi. Le test ELISA, quoique moins sensible que les immunoempreintes et l'IFI, a l'avantage d'offrir une quantification des titres d'auto-anticorps [11]. Dernièrement un test ALBIA (adressable laser bead immunoassay, ou multiplexage) a été développé, offrant, outre une quantification sensible, de par sa nature multiparamétrique, la possibilité, par des panels adaptés, de rechercher certaines des causes de GEM secondaires (lupus, vascularites à ANCA [antineutrophil cytoplasmic antibodies] ou à anticorps anti-GBM [glomerular basement membrane]) [9]. En pratique de routine le test ELISA quantitatif est plus adapté, avec les limites qui sont les siennes, au suivi des patients alors que le test d'IFI plus sensible est indiqué pour le dépistage [11].

\subsection{Le domaine $7 \mathrm{~A}$ de la thrombospondine de type I (THSD7A)}

\subsubsection{Identification de la cible}

Comme pour le PLA2R, I'identification a reposé sur les techniques d'immunoempreintes et de spectrométrie de masse. La THSD7A est une protéine membranaire endothéliale initialement caractérisée au niveau du placenta qui dans le rein se retrouve au pôle basal des podocytes et est absent des glomérules. Sa structure présente des homologies avec le PLA2R: molécule de haut poids moléculaire avec une grande région extracellulaire contenant de nombreux domaines fortement glycosylés par des liaisons de type N. Contrairement au PLA2R, la THSD7A est exprimée sur les podocytes des rongeurs, rendant possible un modèle animal par transfert passif d'auto anticorps humains [7]. Contrairement aux GEM avec anticorps anti-PLA2R, celles avec anticorps anti-THSD7A touchent plus volontiers les femmes [14].

La négativité de 10 à $20 \%$ des GEM idiopathiques pour la recherche des deux auto anticorps (anti-PLA2R et anti-THSD7A) fait évoquer l'existence d'autres cibles auto-antigéniques (alpha énolase, aldose réductase et superoxide dismutase) dont la preuve reste à faire $[14,15]$.

\subsubsection{Les tests diagnostiques}

Comme pour les anticorps anti-PLA2R, le même industriel propose depuis peu un test d'IFI sur cellules transfectées. Le test a été validé chez 1276 patients issus de trois cohortes (une prospective et deux rétrospectives) avec 36 patients ayant des auto anticorps détectables par ce test sur les 39 qui en avaient par immunoempreintes (spécificité de $92 \%$ ) [19].

\subsection{L’immunomarquage surbiopsie rénale} Les GEM associées aux anticorps anti-PLAR2 ou antiTHSD7A peuvent aussi être diagnostiquées par immunofluorescence directe (IFD) ou méthode immuno- 
enzymatique (peroxydase) sur des sections de biopsie rénale incluses en paraffine après digestion à la pronase. Dans les reins de sujets sains, ou affectés de glomérulonéphrites autres que les GEM associées aux anticorps anti-PLAR2 ou anti-THSD7A, le marquage des podocytes est très faible. En cas de GEM primaire par auto-anticorps, on observe un marquage granulaire intense le long des parois capillaires. La différence est cependant plus faible en cas d'anticorps anti-THSD7A, et donc la distinction est plus difficile, requérant un anatomopathologiste expérimenté. En règle générale il existe une bonne corrélation entre le taux d'anticorps circulant et l'intensité du marquage tissulaire [11]. L'analyse combinée de l'intensité du marquage des antigènes podocytaires (PLA2R et THSD7A), du rapport des sousclasses d'IgG (IgG1 versus IgG4) et de la voie d'activation du complément en cause permet de distinguer les GEM primaires des GEM secondaires [14].

\section{L'apport diagnostique de la sérologie auto-immune}

\subsection{L'approche classique}

La ponction biopsie rénale (PBR) est le gold standard pour mettre en évidence les lésions histologiques caractéristiques de GEM.

Toutefois il arrive que faute de matériel suffisant obtenu lors du prélèvement, ou en cas de contreindication à la réalisation d'une PBR, le diagnostic positif ne puisse être affirmé. La présence d'une sérologie positive au sein d'un faisceau d'arguments peut conforter un diagnostic. Un autre défi est de faire la distinction entre GEM primaire et GEM secondaire, plus particulièrement celle associée à une néoplasie chez le sujet âgé. L'occurrence simultanée d'une GEM et d'une néoplasie n'affirme pas systématiquement un lien de causalité et peut relever d'une simple coïncidence. À l'inverse, l'absence d'un cancer documenté n'exclut pas son développement ultérieur dans la progression de la GEM. Dans la pratique, l'enquête étiologique associe l'anamnèse, l'examen clinique, les données de laboratoire et celles d'imagerie. II n'y a pas de consensus sur l'agressivité des investigations, notamment pour identifier un cancer occulte. L'examen de la biopsie rénale peut apporter des indices suggestifs: des dépôts autres que sub-épithéliaux, soit intra-membranaires, voire mésangiaux, des dépôts de C1q, de sous-classes IgG1, IgG2 ou IgG3 sont plus en faveur d'une GEM secondaire [11]. L'absence de dépôts d'IgG4 au diagnostic semble un indicateur fort de survenue ultérieure de cancer [4]

\subsection{L'apport diagnostique \\ des anticorps anti-PLA2R}

La spécificité des anticorps anti-PLA2R semble proche de $100 \%$ [20]. Dans une méta-analyse regroupant 15 études et 2212 patients, la spécificité est de $99 \%$ (95\% Cl 96-100) et la sensibilité de $78 \%$ (95\% Cl 68-87). Ces anticorps ne sont détectés ni chez les patients avec d'autres maladies rénales ou systémiques, ni chez les sujets sains. Cela conduit certains auteurs à proposer que la ponction biopsie rénale ne soit plus systématique, plus particulièrement lorsqu'il existe des contre-indications relatives à sa réalisation [4], ce que contestent d'autres auteurs [15]. Un test positif pour la recherche des anticorps anti-PLA2R se voit dans 50 à $80 \%$ des GEM primaires. La grande variabilité des résultats entre les études relève de plusieurs causes: la nature du test utilisé, avec les immunoempreintes, l'IFI et le multiplexage plus sensibles que le test ELISA, l'origine ethnique, avec une plus faible prévalence dans les études japonaises [21]. Quoi qu'il en soit le paramètre qui contribue le plus à cette variabilité semble être la chronologie de l'enquête sérologique au cours de l'évolution de la maladie. Il existe trois situations pouvant expliquer l'incapacité des tests sérologiques à mettre en évidence des anticorps antiPLA2R au cours d'une authentique GEM auto-immune [11]. Le premier scénario s'explique par la survenue d'une rémission, spontanée ou induite par le traitement immunosuppresseur déjà institué. La rémission immunologique précède toujours la rémission clinique, ce qui peut expliquer la persistance décalée d'une protéinurie, que peut expliquer aussi l'existence de lésions podocytaires irréversibles d'une GEM éteinte. C'est dans ces situations que la recherche par immunomarquage de la ponction biopsie rénale des anticorps anti-PLA2R apporte une plus-value diagnostique: alors que $22 \%$ des sérums de patients sont positifs au cours du suivi, $59 \%$ le sont au diagnostic [22]. Dans le second scénario, au tout début de la maladie, les anticorps circulants sont rapidement épurés de la circulation par fixation aux podocytes; ce n'est que lorsque le rein n'est plus en capacité de jouer son rôle d'éponge que les anticorps sériques deviennent durablement détectables, d'où la recommandation de renouveler la recherche à au moins 3 mois d'intervalle [11]. Dans ces deux situations, qui représentent jusqu'à $20 \%$ des patients séronégatifs, la biopsie rénale objective un marquage glomérulaire par les anticorps anti-PLA2R. Le troisième scénario est celui de la GEM auto-immune médiée par un autre mécanisme, anticorps anti-THSD7A ou cible inconnue. À la question de savoir si la mise en évidence d'anticorps anti-PLA2R dans le sérum d'un patient exclut une GEM secondaire et permet de s'affranchir d'un lourd bilan étiologique, les données de la littérature sont contradictoires [11]. Pour celles qui retrouvent une étiologie, la question est alors de savoir s'il s'agit d'une réelle GEM secondaire ou bien de la simple occurrence simultanée de deux pathologies. La recherche des données histologiques précédemment citées (localisation des dépôts, répartition des sous-classes d'lgG) permet de statuer tout comme l'évolution clinique: la survenue de rémission de la GEM en l'absence de traitement de la maladie secondaire est un argument pour la survenue simultanée des deux, et à l'inverse la persistance de la protéinurie malgré le succès du traitement de la maladie secondaire un argument contre. II existe cependant quelques cas d'authentiques GEM avec colocalisation de dépôts d'anticorps anti-PLA2R et d'antigènes exogènes, notamment viraux (hépatite virale $B$ ou C) [23] suggérant que c'est la maladie rénale qui induit secondairement la réponse anti-PLA2R. 


\subsection{L'apport diagnostique des anticorps anti-THSD7A}

Approximativement 2,5 à $5 \%$ des patients avec une GEM primaire ont des auto anticorps anti-THSD7A, ce qui correspond à 8 à $14 \%$ de ceux qui sont négatifs pour la recherche des anticorps anti-PLA2R [7]. Dans cette étude initiale seuls deux patients avaient une autre pathologie (un lupus systémique et un cancer de la prostate), mais sans les caractéristiques histologiques rénales d'une GEM secondaire et plutôt une prédominance de dépôts d'lgG4, plaidant donc pour la coexistence deux pathologies distinctes. Jusqu'à présent donc, les anticorps anti-THSD7A n'ont pas été détectés chez des sujets sains ou souffrants d'autres pathologies rénales ou systémiques, conduisant à une spécificité de $100 \%$. Le pourcentage de GEM avec anti-THSD7A varie de $3 \%$ en Europe et aux USA à $9 \%$ au Japon [11, 24]. Le fait remarquable de ces études est la découverte dans une proportion non négligeable $(20 \%)$ de néoplasie survenant dans un délai de trois mois après la découverte de la GEM $[19,25]$ : I'expression dans les tumeurs et dans les cellules folliculaires dendritiques des ganglions drainants du mRNA de la THSD7A, et la diminution du titre des anticorps et de la protéinurie après le traitement réussi du cancer plaident pour une rupture de tolérance dans la tumeur aboutissant aux dépôts glomérulaires, ce qui oblige à revoir le concept même de GEM primaire et secondaire.

Une seule publication fait état de la double positivité anti-PLA2R et anti-THSD7A, aussi bien dans le rein que dans le sérum, ce qui pose la question de l'occurrence simultanée des deux pathologies ou bien de celle d'une nouvelle entité. À ce stade on ne sait pas si les deux auto anticorps sont pathogènes, ou bien si l'un induit la survenue de l'autre. L'association préférentielle à l'haplotype HLA DQA1 plaide pour une susceptibilité à l'auto-immunité vis-à-vis des antigènes podocytaires [26].

De même, une seule publication [27] fait état d'un patient se présentant avec un syndrome néphrotique majeur, une glomérulonéphrite rapidement progressive, des lésions histologiques rénales de glomérulonéphrite extra-capillaire et extra-membraneuse associés à des pANCA anti-MPO (myéloperoxidase) et des anticorps anti-PLA2R, en faveur de la survenue simultanée des deux pathologies.

\subsection{Proposition d'algorithme diagnostique}

Certains auteurs proposent donc désormais un algorithme diagnostique intégrant la recherche des auto-anticorps permettant, dans certaines situations bien ciblées, de s'affranchir de la PBR, algorithme qui demande à être validé par de larges études prospectives (figure 1) [11].

\section{L'apport pronostique de la sérologie auto-immune}

\subsection{L'approche classique}

L'évolution naturelle de la GEM est imprévisible et résumée par la règle des trois tiers [15]. Trois devenirs, de proportion grossièrement équivalente, sont observés: rémission spontanée, persistance et stabilité de l'atteinte rénale, progression vers l'insuffisance rénale terminale (IRT). Les deux premiers groupes ne justifient pas la mise en route d'un traitement immunosuppresseur lourd, car non dénué d'effets secondaires toxiques, mais exigent une surveillance clinique stricte à la recherche d'indicateurs de mauvais pronostic qui sont: persistance d'une protéinurie de rang néphrotique pendant plus de six mois, altération rapide de la fonction rénale en six mois ou créatininémie élevée au diagnostic [28]. Le décalage entre la rémission immunologique et la rémission clinique et l'impossibilité prédictive d'une protéinurie persistante entre GEM active et lésions cicatricielles irréversibles rendent difficile la décision d'instaurer un traitement immunosuppresseur. Les auto-anticorps peuvent-ils être une aide $[11,15]$, et sont-ils susceptibles de remettre en cause les algorithmes ayant fait l'objet d'un consensus international [29], qui ne se veut, compte tenu de la spécificité individuelle de chaque patient, qu'une aide à la décision thérapeutique du clinicien, n'ayant pas vocation à se substituer à son jugement [28]?

\subsection{L'apport pronostique}

\section{des anticorps anti-PLA2R}

Comme toute maladie auto-immune la GEM primaire peut se voir comme un iceberg avec trois stades: maladie latente, avec uniquement la présence des auto-anticorps, qu'ils soient pathogènes ou simples marqueurs, maladie silencieuse où en plus des auto-anticorps les lésions histologiques apparaissent sans signes cliniques, et enfin maladie clinique, les $10 \%$ de l'iceberg au-dessus du niveau de la mer, seuls visibles par apparition des signes cliniques [30]. Pour les anticorps anti-PLA2R, les périodes de latence et de silence sont estimées au plus à huit mois [31] et expliquent les données contradictoires de la littérature quant à la corrélation entre titre des auto-anticorps et degré de la protéinurie [11]. En règle générale, plus haut est le titre des auto-anticorps, plus élevé est le risque de progression vers une IRT. Dernièrement, deux publications $[32,33]$ ont identifié deux épitopes conformationnels dominants et recouvrants. En caractérisant les épitopes du PLA2R reconnus par les anticorps des patients à l'aide d'un test ELISA, SeitzPolski et al [34] ont montré que la reconnaissance d'un épitope riche en cystéine était associée avec un fort taux de rémission, alors qu'une dérive épitopique était annonciatrice d'un mauvais pronostic. La disponibilité future de tels outils pourrait faciliter les prises de décision thérapeutique.

\subsection{L'apport pronostique des anticorps anti-THSD7A}

Les données sont beaucoup plus limitées pour les anticorps anti-THSD7A [7, 25]. Elles seraient en faveur du caractère prédictif évolutif du taux des auto-anticorps, mais nécessitent d'être confirmées sur de plus grandes cohortes.

\subsection{Proposition d'algorithme pronostique} Les mêmes auteurs [11] proposent un algorithme décisionnel (figure 2) tenant compte de la positivité de la recherche des auto-anticorps 


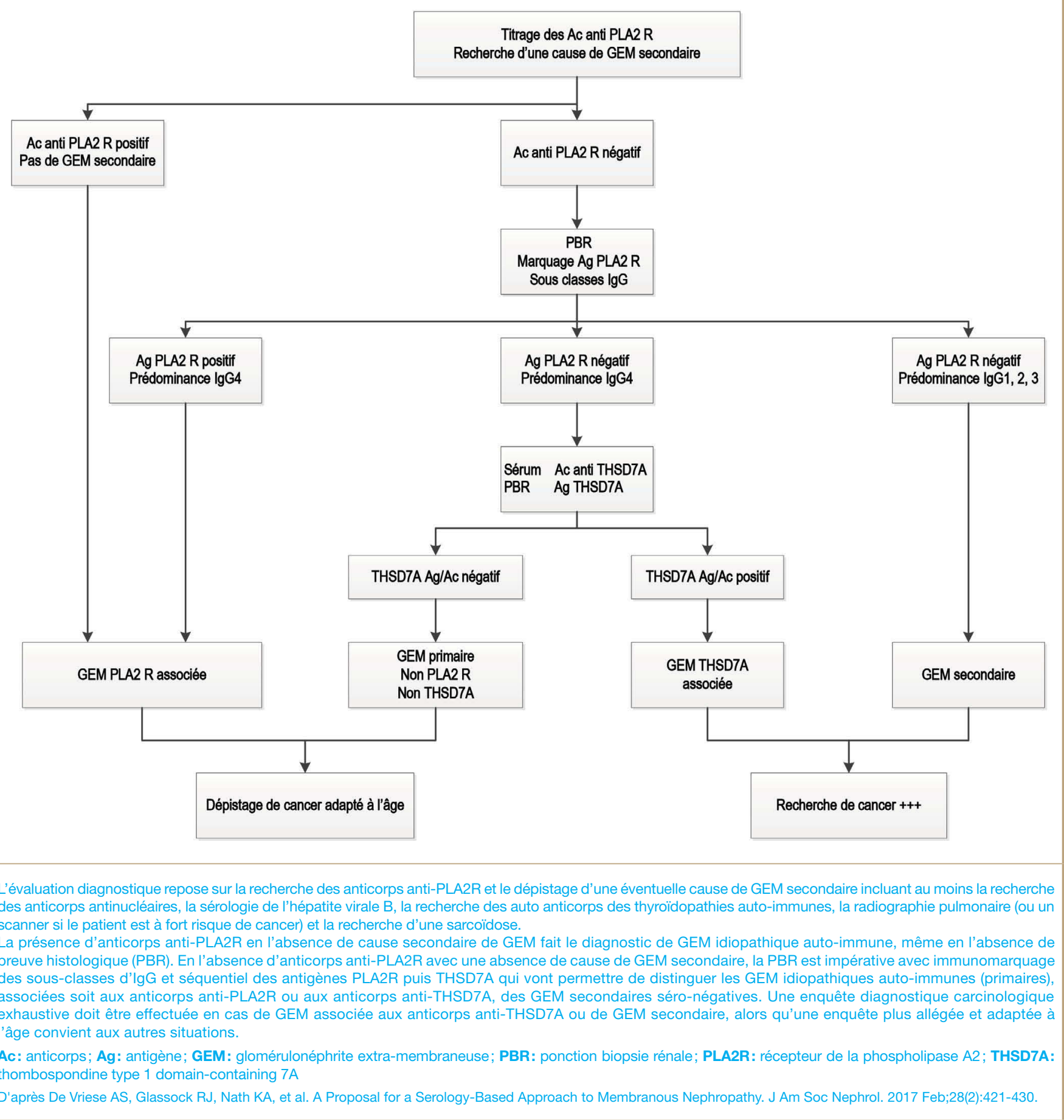

\section{L'apport de la sérologie auto- immune au suivi thérapeutique}

\subsection{L'approche classique}

Les paramètres classiques d'estimation de la fonction rénale (protéinurie, clairance de la créatinine) sont utilisés pour juger de l'efficacité des traitements immunosuppresseurs. Néanmoins ils ne sont pas prédictifs de l'efficacité, une rémission partielle ou complète pouvant s'installer jusqu'à 18 mois après l'achèvement de la cure d'immunosuppresseurs, temps requis pour que le remodelage des podocytes restaure une filtration glomérulaire opérationnelle. On estime qu'environ $20 \%$ des patients traités n'auront jamais de rémission à 10 ans, et que $75 \%$ d'entre eux progresseront vers I'IRT [35]. La biopsie rénale a toute son utilité en objectivant soit des lésions florides, nécessitant la reprise d'un traitement immunosuppresseur, soit des lésions cicatricielles irréversibles relevant d'une autre prise en charge [21]. 


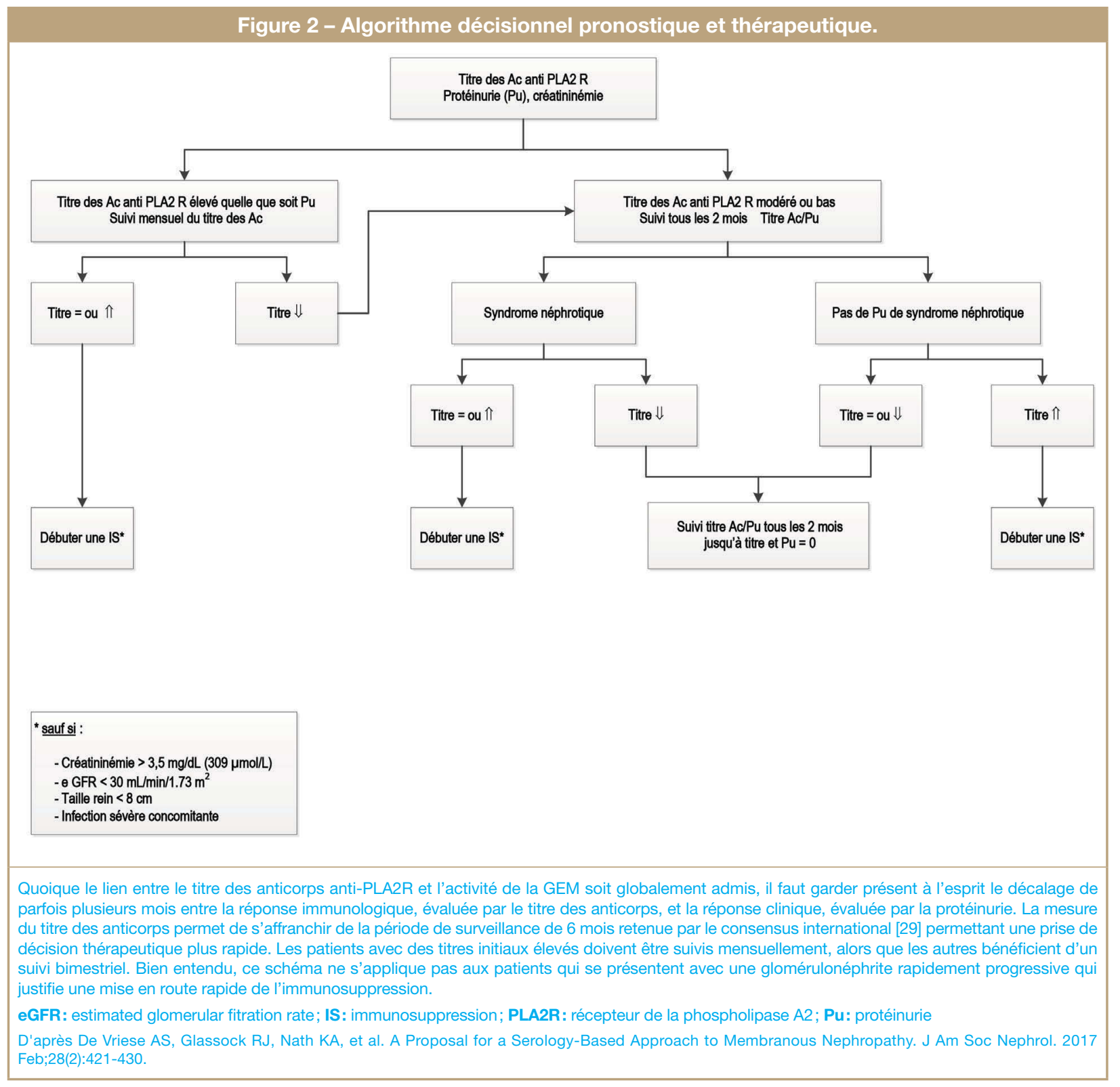

\subsection{Suivi thérapeutique}

\section{par les anticorps anti-PLA2R}

Pour ce qui est du caractère prédictif évolutif du titre des anticorps au diagnostic, les données de la littérature sont contradictoires [11]. Ce qui est néanmoins attesté, c'est la précession de la chute du titre des anticorps par rapport au déclin de la protéinurie: les auto-anticorps chutent rapidement les trois premiers mois du traitement pour disparaître dans un délai de six à neuf mois alors que la disparition de la protéinurie demande 12 à 24 mois, ce qui traduit l'extinction rapide de l'activité immunologique suivie par la restauration tissulaire [15]. De même, la persistance d'auto-anticorps après l'achèvement de la cure d'immunosuppresseurs est un facteur prédictif de rechute dans les deux ans [36]: $58 \%$ des patients sans anticorps détectables après la fin du traitement avaient une rémission persistante à cinq ans alors que tous les patients séropositifs en fin de traitement avaient rechuté. La nature de la drogue ou des combinaisons utilisées (tacrolimus, rituximab, cyclophosphamide) ne semble pas influer sur la cinétique des anticorps. La persistance d'anticorps à titre élevé chez les patients résistants à toute immunosuppression est le rationnel d'utilisation d'échanges plasmatiques dans une étude pilote prometteuse [37].

Le caractère récent de l'identification des anticorps antiTHSD7A ne permet pas d'avoir ce type de données sur leur utilité dans le suivi thérapeutique.

\subsection{Proposition d'algorithme de suivi thérapeutique}

Les mêmes auteurs [11] proposent un algorithme décisionnel (figure 3) tenant compte du titrage des anticorps pour adapter l'immunosuppression. 


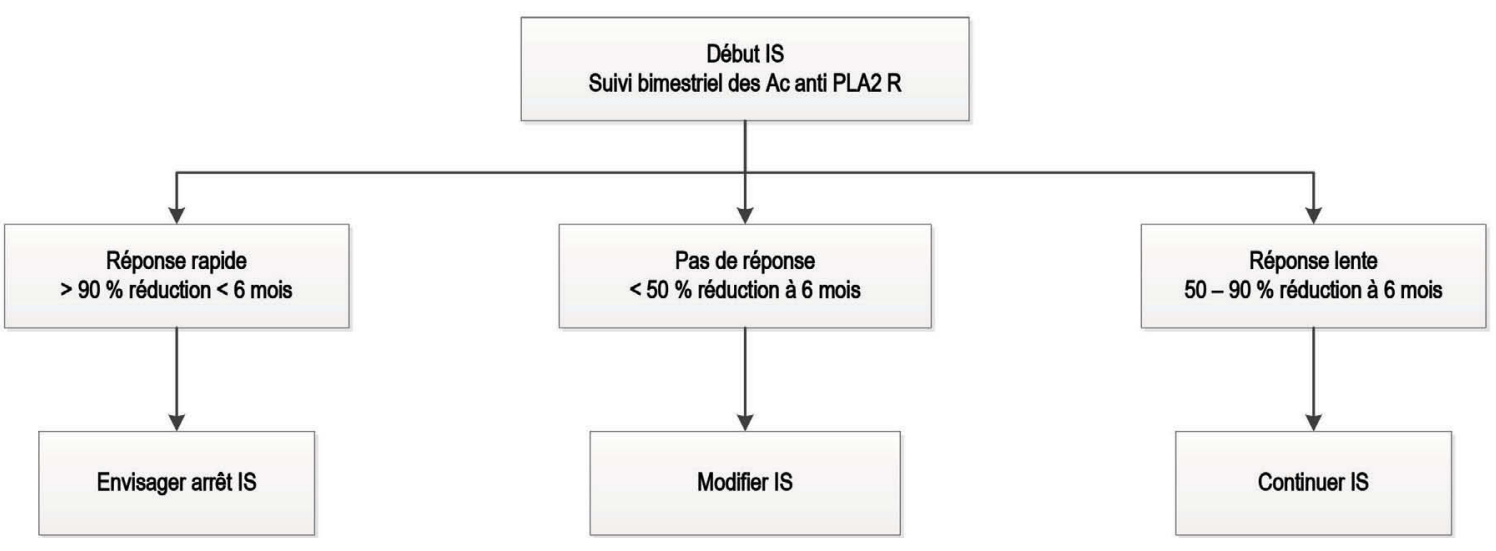

Les patients qui ont une réponse rapide et intense peuvent recevoir une cure d'immunosuppression plus courte que celle recommandée usuellement. II est important de garder présent à l'esprit que l'inhibition de la réponse des lymphocytes dure bien au-delà du traitement, non seulement pour le rituximab, mais aussi pour le cyclophosphamide. Le changement d'immunosuppresseur doit s'envisager en cas de non réponse à six mois. Les patients avec des titres élevés au diagnostic peuvent requérir une cure d'immunosuppresseur plus intense et plus longue, au besoin complétée par des échanges plasmatiques.

IS: immunosuppression; PLA2R: récepteur de la phospholipase A2

D'après De Vriese AS, Glassock RJ, Nath KA, et al. A Proposal for a Serology-Based Approach to Membranous Nephropathy. J Am Soc Nephrol. 2017 Feb;28(2):421-430.

\section{L’apport de la sérologie auto-immune au depistage des recidives post-transplantation}

\subsection{L'approche classique}

Le taux de récidive est estimé de 10 à $40 \%$ [4] si l'on tient compte des formes histologiques subcliniques. Deux pics sont observés: la première année par échec primaire de l'immunosuppression, et après 4 à 5 ans lors de la diminution graduelle du traitement anti-rejet. Un tiers des patients avec une récidive ne progresseront pas vers I'IRT et ne nécessiteront pas la réintroduction d'un traitement immunosuppresseur. II n'existe pas de paramètres cliniques prédictifs de récidive post-transplantation et les données histologiques sont d'interprétation difficile entre atteinte lésionnelle et médicamenteuse [11].

\subsection{Suivi post-transplantation \\ par les anticorps anti-PLA2R}

Là encore les données de la littérature sont contradictoires concernant le caractère prédictif de récidive posttransplantation d'un titre élevé des anticorps avant la transplantation [11]. Pour l'absence de récidive malgré la persistance d'un titre conséquent d'auto-anticorps, les auteurs évoquent la différence des épitopes du PLA2R entre le donneur et le receveur, le greffon n'exprimant pas les épitopes reconnus par les anticorps du receveur. Pour ce qui est des récidives chez des patients séro-négatifs, il est évoqué une GEM non-PLA2R que pourrait objectiver l'immunomarquage de la ponction biopsie rénale. II semble cependant désormais admis que la persistance ou la réapparition des anticorps antiPLA2R sont étroitement associées avec une récidive post-transplantation. Les résultats de différents proto- coles d'étude (PRAM-KT) permettront de mieux préciser les modalités du suivi et les indications thérapeutiques chez le transplanté rénal.

Le suivi de la reconstitution des lymphocytes $B$ circulants au cours du traitement par rituximab participe aussi à l'élaboration de la stratégie thépeutique.

\subsection{Suivi post-transplantation par les anticorps anti-THSD7A}

Des données préliminaires vont dans le même sens pour les anticorps anti-THSD7A [8].

\subsection{Proposition d'algorithme de suivi post-transplantation}

Un suivi régulier des anticorps (bimensuel la première année, annuellement après et chaque fois que la protéinurie réapparaît) peut aider la décision de réintroduire le rituximab [11].

\section{Conclusion}

Les avancées récentes dans la compréhension des mécanismes physiopathologiques des GEM idiopathiques ont induit un changement de paradigme pour le diagnostic et le suivi des patients $[4,11,15]$. La disponibilité des tests diagnostiques doit permettre l'introduction raisonnée de la sérologie auto-immune dans les algorithmes décisionnels.

Liens d'intérêts: les auteurs déclarent ne pas avoir de liens d'intérêts avec le contenu de cet article.

\section{Remerciements}

à Caroline Laval pour l'assistance graphique 


\section{Références}

[1] Herrmann SM, Sethi S, Fervenza FC. Membranous nephropathy: the start of a paradigm shift. Curr Opin Nephrol Hypertens. 2012 Mar; 21(2):203-10.

[2] Callard $\mathrm{P}$, Colombat $\mathrm{M}$. Glomérulonéphrites extra-membraneuses in Noel LH (ed) Atlas de pathologie rénale. Médecine-Sciences Flammarion Paris, 2008: 165-180

[3] Beck LH Jr, Salant DJ. Membranous nephropathy: recent travels and new roads ahead. Kidney Int. 2010 May;77(9):765-70.

[4] Ronco P, Debiec H. Pathophysiological advances in membranous nephropathy: time for a shift in patient's care. Lancet. 2015 May 16;385(9981):1983-92.

[5] Beck LH, Bonegio RGB, Lambeau G, et al., M-type phospholipase A2 receptor as target antigen in idiopathic membranous nephropathy. $\mathrm{N}$ Engl J Med 2009;361(1):11-21.

[6] Augusto JF, Subra JF, Chevailler A. Apport de l'immunologie au diagnostic en néphrologie: actualités en 2012. Revue Francophone des Laboratoires 2012, 444 : 95-96

[7] Tomas NM, Beck LH Jr, Meyer-Schwesinger C, et al. Thrombospondin type-1 domain-containing $7 \mathrm{~A}$ in idiopathic membranous nephropathy. $\mathrm{N}$ Engl J Med. 2014 Dec 11;371(24):2277-87.

[8] Tomas NM, Hoxha E, Reinicke AT, et al. Autoantibodies against thrombospondin type 1 domain-containing $7 \mathrm{~A}$ induce membranous nephropathy. J Clin Invest. 2016 Jul 1;126(7):2519-32.

[9] Behnert A, Schiffer M, Müller-Deile J, et al. Antiphospholipase A2 receptor autoantibodies: a comparison of three different immunoassays for the diagnosis of idiopathic membranous nephropathy. J Immunol Res. 2014; 2014:143274.

[10] Glassock RJ. Antiphospholipase A2 receptor autoantibody guided diagnosis and treatment of membranous nephropathy: a new personalized medical approach. Clin J Am Soc Nephrol. 2014 Aug 7;9(8):1341-3. [11] De Vriese AS, Glassock RJ, Nath KA, et al. A Proposal for a Serology-Based Approach to Membranous Nephropathy. J Am Soc Nephrol. 2017 Feb;28(2):421-430.

[12] Ronco P, Debiec H. Anti-phospholipase A2 receptor antibodies and the pathogenesis of membranous nephropathy. Nephron Clin Pract. 2014;128(3-4):232-7.

[13] Hanasaki K. Mammalian phospholipase A2: phospholipase A2 receptor. Biol Pharm Bull. 2004 Aug;27(8):1165-7.

[14] Cattran DC, Brenchley PE. Membranous nephropathy: integrating basic science into improved clinical management. Kidney Int. 2017 Mar;91(3):566-574.

[15] Obrisca B, Ismail G, Jurubita R, et al. Antiphospholipase A2 Receptor Autoantibodies: A Step Forward in the Management of Primary Membranous Nephropathy. Biomed Res Int. 2015; 2015:249740. doi: 10.1155/2015/249740. Epub 2015 Oct 20

[16] Liu YH, Chen $\mathrm{CH}$, Chen SY, et al. Association of phospholipase A2 receptor 1 polymorphisms with idiopathic membranous nephropathy in Chinese patients in Taiwan. J Biomed Sci. 2010 Oct 11; 17:81.

[17] Stanescu HC, Arcos-Burgos M, Medlar A, et al Risk HLA-DQA1 and PLA(2)R1 alleles in idiopathic membranous nephropathy. N Engl J Med. 2011 Feb 17;364(7):616-26.

[18] Hofstra JM, Wetzels JF. Anti-PLA 2R antibodies in membranous nephropathy: ready for routine clinical practice? Neth J Med. 2012 Apr;70(3):109-13

[19] Hoxha E, Beck LH Jr, Wiech T, et al. An Indirect Immunofluorescence Method Facilitates Detection of Thrombospondin Type 1 DomainContaining 7A-Specific Antibodies in Membranous Nephropathy. J Am Soc Nephrol. 2017 Feb;28(2):520-531.
[21] Du Y, Li J, He F, et al. The diagnosis accuracy of PLA2R-AB in the diagnosis of idiopathic membranous nephropathy: a meta-analysis. [20] Dai H, Zhang H, He Y. Diagnostic accuracy of PLA2R autoantibodies and glomerular staining for the differentiation of idiopathic and secondary membranous nephropathy: an updated meta-analysis. Sci Rep. 2015 Mar 5; 5:8803.

[22] Svobodova B, Honsova E, Ronco P, et al. Kidney biopsy is a sensitive tool for retrospective diagnosis of PLA2R-related membranous nephropathy. Nephrol Dial Transplant. 2013 Jul; 28(7):1839-44.

[23] Xie Q, Li Y, Xue J, Xiong Z, et al. Renal phospholipase A2 receptor in hepatitis B virus-associated membranous nephropathy. Am J Nephrol. 2015;41(4-5):345-53.

[24] Iwakura T, Ohashi N, Kato A, et al. Prevalence of Enhanced Granular Expression of Thrombospondin Type-1 Domain-Containing $7 \mathrm{~A}$ in the Glomeruli of Japanese Patients with Idiopathic Membranous Nephropathy. PLoS One. 2015 Sep 22; 10(9):e0138841.

[25] Hoxha E, Wiech T, Stahl PR, et al. A Mechanism for CancerAssociated Membranous Nephropathy. N Engl J Med. 2016 May 19;374(20):1995-6.

[26] Larsen CP, Cossey LN, Beck LH. THSD7A staining of membranous glomerulopathy in clinical practice reveals cases with dual autoantibody positivity. Mod Pathol. 2016 Apr;29(4):421-6.

[27] Surindran S, Ayalon R, Hasan N, et al. Coexistence of ANCAassociated glomerulonephritis and anti-phospholipase $A(2)$ receptor antibody-positive membranous nephropathy. Clin Kidney J. 2012 Apr;5(2):162-165.

[28] Alfaadhel T, Cattran D. Management of Membranous Nephropathy in Western Countries. Kidney Dis (Basel). 2015 Sep;1(2):126-37.

[29] Kidney Disease: Improving Global Outcomes (KDIGO) Glomerulonephritis Work Group. KDIGO Clinical Practice Guideline for Glomérulonephritis. Kidney Int Suppl 2012; 2:139-274.

[30] Whittingham S, Mackay IR. The iceberg analogy of autoimmunity. Postgrad Med J. 1972 Feb;48(556):95-7.

[31] Guerry MJ, Vanhille P, Ronco P, et al. Serum anti-PLA2R antibodies may be present before clinical manifestations of membranous nephropathy. Kidney Int. 2016 Jun;89(6):1399.

[32] Kao L, Lam V, Waldman M, et al. Identification of the immunodominant epitope region in phospholipase A2 receptor-mediating autoantibody binding in idiopathic membranous nephropathy. J Am Soc Nephrol. 2015 Feb;26(2):291-301.

[33] Fresquet M, Jowitt TA, Gummadova J, et al. Identification of a major epitope recognized by PLA2R autoantibodies in primary membranous nephropathy. J Am Soc Nephrol. 2015 Feb;26(2):302-13

[34] Seitz-Polski B, Dolla G, Payré C, et al. Epitope Spreading of Autoantibody Response to PLA2R Associates with Poor Prognosis in Membranous Nephropathy. J Am Soc Nephrol. 2016 May;27(5): 1517-33.

[35] Kanigicherla DA, Short CD, Roberts SA, et al. Long-term outcomes of persistent disease and relapse in primary membranous nephropathy. Nephrol Dial Transplant. 2016 Dec;31(12):2108-2114.

[36] Bech AP, Hofstra JM, Brenchley PE, et al. Association of antiPLA2R antibodies with outcomes after immunosuppressive therapy in idiopathic membranous nephropathy. Clin J Am Soc Nephrol. 2014 Aug 7;9(8):1386-92.

[37] Müller-Deile J, Schiffer L, Hiss M, Haller H, Schiffer M. A new rescue regimen with plasma exchange and rituximab in high-risk membranous glomerulonephritis. Eur J Clin Invest. 2015 Dec;45(12):1260-9. 